\title{
Vertebral aneurysm due to isolated spontaneous dissection of the intracranial vertebral artery
}
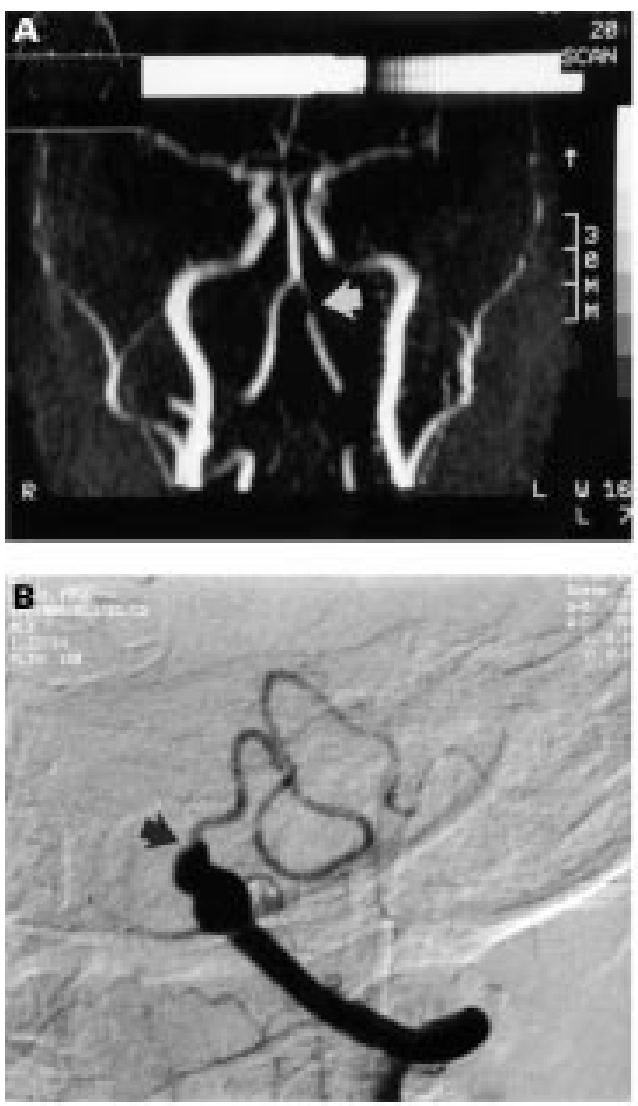

A 31 year old man developed left sided headache followed three days later by a severe exacerbation with vomiting. There was no antecedent head or neck trauma and no neurological signs were present. CT and CSF analysis disclosed no evidence of subarachnoid haemorrhage.

Magnetic resonance angiography (figure A) disclosed an isolated stenotic segment of the lett intracranial vertebral artery (ICVA) (arrow). Four weeks later intra-arterial angiography was performed. The left VA injection (figure B) showed aneurysmal dilatation of the ICVA with the left posterior inferior cerebellar artery (PICA) arising from the most rostral portion of the aneurysm (arrow). Distal to this, the ICVA was occluded. On the right VA injection (figure C) a small amount of reflux was seen into a remnant stump of the left terminal ICVA (arrow), but no filling of the left PICA was seen. T1 weighted MRI (figure D) showed high signal in the wall of the left ICVA due to haemorrhage (arrow), the characteristic appearance of a dissection.
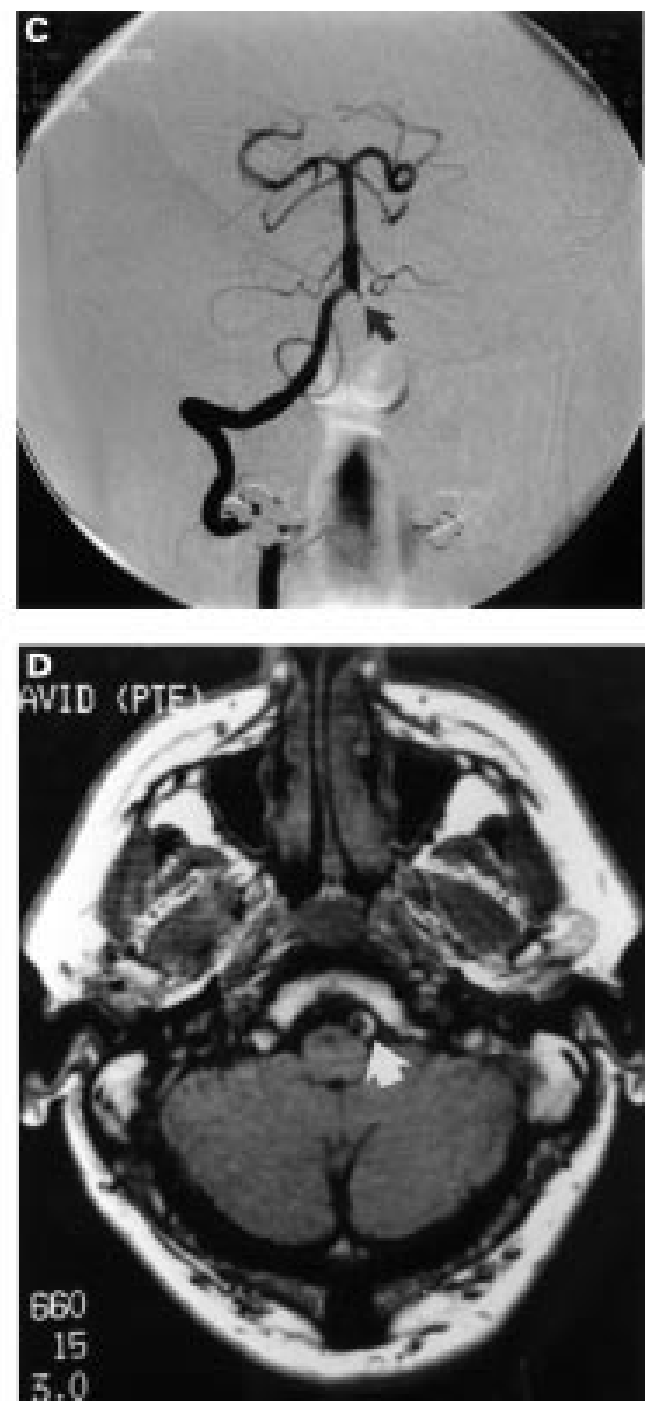

Management was conservative given the fear of sacrificing the left PICA in an interventional procedure, and the absence of evidence that the aneurysm had bled.

P J MARTIN T P ENEVOLDSON Department of Neurology, Walton Centre for Neurology and Neurosurgery, Lower Lane, Liverpool L9 7AL UK

Correspondence to: Dr PJ Martin, Department of Neurology, Walton Centre for Neurology and Neurosurgery, Lower Lane, Liverpool L9 7AL UK. 